\title{
A Statistical Study of Three Major Crops of India
}

\author{
Rajani Devi \\ Assistant Professor \\ University College for Women \\ Hyderabad
}

\author{
V. V. Haragopal, PhD \\ Professor \\ Osmania University \\ Hyderabad
}

\begin{abstract}
Agriculture is largest and important sector of Indian Economy. The major objective of this paper is to examine the production growth of food grains for different seasons for the sustainability of agricultural sector in the next decade. The analytics considers the data for the period of 2003-2016. The major conclusions drawn from this paper is that the various crop productions show that a major increase almost in total output over the years. This paper recommends some measures as a positive step to improve the production of crop of the agricultural sector in India.
\end{abstract}

\section{Keywords}

Major crops, Rice, Jowar and Maize ,Analysis of variance, Most common hoc tests ,Regression, Statistical significance.

\section{INTRODUCTION}

Agriculture is the largest and an important sector in Indian economy. It is indispensible for the sustenance and growth of Indian economy. India is a large producer of several agricultural products. In terms of quantity of production, India is the second largest producer in jowar and rice. In this study, the objective is to study major agricultural crop production(Ajit Kaur Bhatia, Rajender Prasad, S.K Shama and Rajinder Kaur (2005);J S Dhekale, Rajendar Prasad and V K Gupta (2003)). The secondary source data is used to see if any pattern can be observed and is studied by analyzing the data with some popular statistical techniques. (Mann, Prem S (1995);Trochim, William M.K (2006);Jaccard. J; Becker, M.A.Wood.G (1984);:Bailey R.A (2008);Cochran, William G; Cox, Gertrude M (1992);Ronald A Fisher(1954);Ajit Kaur Bhatia, Rajender Prasad, S.K Shama and Rajinder Kaur (2005);J S Dhekale, Rajendar Prasad and V K Gupta (2003)). Three major agricultural crops, viz., Rice, Jowar and Maize have been considered under Rabi and Kharif seasons

\section{DATA DESCRIPTION AND SOURCE OF DATA}

Here, the Secondary data is collected from Agricultural Statistics Division, Directorate of Economics and Statistics, Department of Agriculture, Cooperation and Farmers welfare. The data is collected for the years 2003-2016 for three major crops rice, jowar and maize for two seasons Rabi and Kharif.

Table 1: Production in million tones

\begin{tabular}{|c|c|c|c|c|c|c|c|c|}
\hline Crop & Season & $\begin{array}{c}\mathbf{2 0 0 3 -} \\
\mathbf{0 4}\end{array}$ & $\begin{array}{c}\text { 2004- } \\
\mathbf{0 5}\end{array}$ & $\begin{array}{c}\mathbf{2 0 0 5 -} \\
\mathbf{0 6}\end{array}$ & $\begin{array}{c}\mathbf{2 0 0 6} \\
\mathbf{0 7}\end{array}$ & $\begin{array}{c}\mathbf{2 0 0 7 -} \\
\mathbf{0 8}\end{array}$ & $\begin{array}{c}\mathbf{2 0 0 8}- \\
\mathbf{0 9}\end{array}$ & $\begin{array}{c}\mathbf{2 0 0 9} \\
\mathbf{1 0}\end{array}$ \\
\hline Rice & Kharif & 78.62 & 72.23 & 78.27 & 80.17 & 82.66 & 84.91 & 75.92 \\
\hline & Rabi & 9.91 & 10.90 & 13.52 & 13.18 & 14.03 & 14.27 & 13.18 \\
\hline Jowar & Kharif & 4.84 & 4.04 & 4.07 & 3.71 & 4.11 & 3.05 & 2.76 \\
\hline & Rabi & 1.84 & 3.20 & 3.56 & 3.44 & 3.81 & 4.19 & 3.93 \\
\hline Maize & Kharif & 12.73 & 11.48 & 12.16 & 11.56 & 15.11 & 14.12 & 12.29 \\
\hline & Rabi & 2.25 & 2.70 & 2.55 & 3.54 & 3.85 & 5.61 & 4.43 \\
\hline
\end{tabular}

Table 1: Production in million tones (continued)

\begin{tabular}{|c|c|c|c|c|c|c|c|}
\hline Crop & Season & $\begin{array}{c}\mathbf{2 0 1 0 -} \\
\mathbf{1 1}\end{array}$ & $\begin{array}{c}\mathbf{2 0 1 1 -} \\
\mathbf{1 2}\end{array}$ & $\begin{array}{c}\mathbf{2 0 1 2 -} \\
\mathbf{1 3}\end{array}$ & $\begin{array}{c}\mathbf{2 0 1 3 -} \\
\mathbf{1 4}\end{array}$ & $\begin{array}{c}\mathbf{2 0 1 4}-\mathbf{1 5} \\
\mathbf{2 0 1 5 -} \\
\mathbf{1 6}\end{array}$ \\
\hline Rice & Kharif & 80.65 & 92.78 & 92.37 & 91.50 & 91.39 & 90.59 \\
\hline & Rabi & 15.33 & 12.52 & 12.87 & 15.15 & 14.09 & 12.77 \\
\hline Jowar & Kharif & 3.44 & 3.29 & 2.84 & 2.39 & 2.30 & 1.87 \\
\hline & Rabi & 3.56 & 2.69 & 2.44 & 3.15 & 3.15 & 2.72 \\
\hline
\end{tabular}




\begin{tabular}{|l|c|c|c|c|c|c|c|}
\hline Maize & Kharif & 16.64 & 16.49 & 16.19 & 17.14 & 17.01 & 15.50 \\
\hline & Rabi & 5.09 & 5.27 & 6.06 & 7.11 & 7.16 & 5.53 \\
\hline
\end{tabular}

\section{DATA ANALYSIS OF THE DATA}

\subsection{Descriptive Statistics}

Descriptive Statistics are used to describe the basic features of the data in a study. They provide simple summaries about the sample and the measures. Descriptive Statistics provides a quick method to make comparisons between different data sets and to spot the smallest and largest values and trends or changes over a period of time.In the data set various descriptive comparisons have made for three major crops rice, jowar and maize during Rabi and Kharif. These measures show what value is in the middle of this data? And where does

a particular data value stand with respect, with the other values in the data set? For instance in the table mean Rabi rice production is 13.2092 million tones. This single number describes that the estimated production in any year should be around 13.2092 million tones. Dispersion refers to the spread of the values around the central tendency (mean).There are two common measures of dispersion, Range and Standard deviation. Range is simply the highest value minus lowest value. For the above data, for instance, for Rabi season the rice production has the highest production which is 15.33 tones and lowest production is 9.91 tones. So for Rabi season the rice production range is15.33-9.91=5.42. The standard deviation is a more accurate and detailed estimate of dispersion. The standard deviation shows the relation that set of production values to the mean of the sample. The standard deviation allows us to reach some conclusions about specific production values in our distribution (Table 2).

Table 2: Descriptives

\begin{tabular}{|c|c|c|c|c|}
\hline & $\mathbf{N}$ & Mean & $\begin{array}{c}\text { Standard } \\
\text { Deviation }\end{array}$ & Std.Error \\
\hline Rabi_rice & 13 & 13.2092 & 1.5282 & 0.42319 \\
\hline Kharif_rice & 13 & 84.0123 & 7.05369 & 1.95634 \\
\hline Rabi_jowar & 13 & 3.2062 & 0.65346 & 0.18124 \\
\hline Kharif_jowar & 13 & 3.2854 & 0.85596 & 0.23740 \\
\hline Rabi_maize & 13 & 4.7038 & 1.64492 & 0.45662 \\
\hline Kharif_maize & 13 & 13.9554 & 3.35836 & 0.93144 \\
\hline Total & 78 & 20.3954 & 29.16088 & 3.30182 \\
\hline
\end{tabular}

Table 2: Descriptives(continued)

\begin{tabular}{|c|c|c|c|}
\hline \multicolumn{2}{|c|}{$\begin{array}{c}\text { 95\% Confidence Interval for } \\
\text { Mean }\end{array}$} & Minimum & Maximum \\
\cline { 1 - 2 } $\begin{array}{c}\text { Lower } \\
\text { Bound }\end{array}$ & Upper Bound & & \\
\hline 12.2872 & 14.1313 & 9.91 & 15.33 \\
\hline 79.7498 & 88.2748 & 72.23 & 92.78 \\
\hline 2.8113 & 3.6010 & 1.84 & 4.19 \\
\hline
\end{tabular}

\begin{tabular}{|c|c|c|c|}
\hline 2.7681 & 3.8026 & 1.87 & 4.84 \\
\hline 3.7098 & 5.6979 & 2.25 & 7.16 \\
\hline 11.9259 & 15.9848 & 5.16 & 17.14 \\
\hline 13.8206 & 26.9701 & 1.84 & 92.78 \\
\hline
\end{tabular}

\subsection{One Way ANOVA}

The overall ANOVA is seen for all crops Rabi and Kharif seasons the Rice, Jowar and Maize production there is more significant difference between the groups of Rabi and kharif of Rice , Jowar and Maize crops indicating that for all the years the average production is not the same and has variations indicating that there is some sort of environmental and other climatic changes show this variation (table 3 ).

Table 3:ANOVA

rabi_rice

\begin{tabular}{|c|c|c|c|c|c|}
\hline & $\begin{array}{c}\text { Sum of } \\
\text { Squares }\end{array}$ & df & $\begin{array}{c}\text { Mean } \\
\text { Square }\end{array}$ & F & Sig. \\
\hline Between & 64670.755 & 5 & 12934.15 & 1154. & .000 \\
Groups & 806.721 & 72 & 11.204 & & \\
Within & & & & & \\
Groups & 65477.476 & 77 & & & \\
Total & & & & & \\
\end{tabular}

\subsection{Post Hoc Tests}

From Table 4 given below, it is clear that Post hoc analysis is a step wise multiple comparisons procedure used to identify sample means that are significantly different from each other .Post hoc test is used whenever a significant difference between three or more sample means has been revealed by an analysis of variance (ANOVA) test .The most common hoc tests are Tukey HSD, Scheff, LSD (Fisher's least significant difference), Bonferroni, Dunnett t (2 sided) tests. All these tests are used to determine whether any of the differences between means are statistically different. These tests are used to find out which pairs of means are significant. To determine the difference the $\mathrm{p}$ value is tested at 5\% los (significant value) to assess the null hypothesis. The null hypothesis states that all means are equal usually a significant level (denoted as $\mathrm{p}$ or alpha) of 0.05 works well. If $\mathrm{p}>0.05$ then there is no significant difference between means of different groups. In the above table 3 the mean differences with the symbol * has significant difference between group means. 


\begin{tabular}{|c|c|c|c|c|c|c|c|}
\hline \multicolumn{8}{|c|}{ Table 4 :Multiple Comparisons } \\
\hline \multicolumn{8}{|c|}{ Dependent Variable: rabi_rice } \\
\hline & \multirow[t]{2}{*}{ (I) target } & \multirow[t]{2}{*}{ (J) target } & \multirow{2}{*}{$\begin{array}{c}\text { Mean Difference } \\
(\mathbf{I}-\mathbf{J}) \\
\end{array}$} & \multirow[t]{2}{*}{ Std. Error } & \multirow[t]{2}{*}{ Sig. } & \multicolumn{2}{|c|}{$\mathbf{9 5 \%}$ Confidence Interval } \\
\hline & & & & & & Lower Bound & Upper Bound \\
\hline \multirow{30}{*}{ Tukey HSD } & \multirow{5}{*}{ Rabi_rice } & Kharif_rice & $-70.80308^{*}$ & 1.31292 & .000 & -74.6471 & -66.9590 \\
\hline & & rabi_jowar & $10.00308^{*}$ & 1.31292 & .000 & 6.1590 & 13.8471 \\
\hline & & kharif_jowar & $9.92385^{*}$ & 1.31292 & .000 & 6.0798 & 13.7679 \\
\hline & & rabi_maize & $8.50538^{*}$ & 1.31292 & .000 & 4.6613 & 12.3494 \\
\hline & & kharif_maize & -.74615 & 1.31292 & .993 & -4.5902 & 3.0979 \\
\hline & \multirow{5}{*}{ Kharif_rice } & Rabi_rice & $70.80308^{*}$ & 1.31292 & .000 & 66.9590 & 74.6471 \\
\hline & & rabi_jowar & $80.80615^{*}$ & 1.31292 & .000 & 76.9621 & 84.6502 \\
\hline & & kharif_jowar & $80.72692^{*}$ & 1.31292 & .000 & 76.8829 & 84.5710 \\
\hline & & rabi_maize & $79.30846^{*}$ & 1.31292 & .000 & 75.4644 & 83.1525 \\
\hline & & kharif_maize & $70.05692^{*}$ & 1.31292 & .000 & 66.2129 & 73.9010 \\
\hline & \multirow{5}{*}{ rabi_jowar } & Rabi_rice & $-10.00308^{*}$ & 1.31292 & .000 & -13.8471 & -6.1590 \\
\hline & & Kharif_rice & $-80.80615^{*}$ & 1.31292 & .000 & -84.6502 & -76.9621 \\
\hline & & kharif_jowar & -.07923 & 1.31292 & 1.000 & -3.9233 & 3.7648 \\
\hline & & rabi_maize & -1.49769 & 1.31292 & .863 & -5.3417 & 2.3464 \\
\hline & & kharif_maize & $-10.74923^{*}$ & 1.31292 & .000 & -14.5933 & -6.9052 \\
\hline & \multirow{5}{*}{$\begin{array}{c}\text { kharif_jow } \\
\text { ar }\end{array}$} & Rabi_rice & $-9.92385^{*}$ & 1.31292 & .000 & -13.7679 & -6.0798 \\
\hline & & Kharif_rice & $-80.72692^{*}$ & 1.31292 & .000 & -84.5710 & -76.8829 \\
\hline & & rabi_jowar & .07923 & 1.31292 & 1.000 & -3.7648 & 3.9233 \\
\hline & & rabi_maize & -1.41846 & 1.31292 & .888 & -5.2625 & 2.4256 \\
\hline & & kharif_maize & $-10.67000^{*}$ & 1.31292 & .000 & -14.5141 & -6.8259 \\
\hline & \multirow{5}{*}{ rabi_maize } & Rabi_rice & $-8.50538^{*}$ & 1.31292 & .000 & -12.3494 & -4.6613 \\
\hline & & Kharif_rice & $-79.30846^{*}$ & 1.31292 & .000 & -83.1525 & -75.4644 \\
\hline & & rabi_jowar & 1.49769 & 1.31292 & .863 & -2.3464 & 5.3417 \\
\hline & & kharif_jowar & 1.41846 & 1.31292 & .888 & -2.4256 & 5.2625 \\
\hline & & kharif_maize & $-9.25154^{*}$ & 1.31292 & .000 & -13.0956 & -5.4075 \\
\hline & \multirow{5}{*}{$\begin{array}{c}\text { kharif_mai } \\
\text { ze }\end{array}$} & Rabi_rice & .74615 & 1.31292 & .993 & -3.0979 & 4.5902 \\
\hline & & Kharif_rice & $-70.05692^{*}$ & 1.31292 & .000 & -73.9010 & -66.2129 \\
\hline & & rabi_jowar & $10.74923^{*}$ & 1.31292 & .000 & 6.9052 & 14.5933 \\
\hline & & kharif_jowar & $10.67000^{*}$ & 1.31292 & .000 & 6.8259 & 14.5141 \\
\hline & & rabi_maize & $9.25154^{*}$ & 1.31292 & .000 & 5.4075 & 13.0956 \\
\hline \multirow{8}{*}{ Scheffe } & \multirow{5}{*}{ Rabi_rice } & Kharif_rice & $-70.80308^{*}$ & 1.31292 & .000 & -75.2957 & -66.3104 \\
\hline & & rabi_jowar & $10.00308^{*}$ & 1.31292 & .000 & 5.5104 & 14.4957 \\
\hline & & kharif_jowar & $9.92385^{*}$ & 1.31292 & .000 & 5.4312 & 14.4165 \\
\hline & & rabi_maize & $8.50538^{*}$ & 1.31292 & .000 & 4.0127 & 12.9980 \\
\hline & & kharif_maize & -.74615 & 1.31292 & .997 & -5.2388 & 3.7465 \\
\hline & \multirow{3}{*}{ Kharif_rice } & Rabi_rice & $70.80308^{*}$ & 1.31292 & .000 & 66.3104 & 75.2957 \\
\hline & & rabi_jowar & $80.80615^{*}$ & 1.31292 & .000 & 76.3135 & 85.2988 \\
\hline & & kharif_jowar & $80.72692^{*}$ & 1.31292 & .000 & 76.2343 & 85.2196 \\
\hline
\end{tabular}




\begin{tabular}{|c|c|c|c|c|c|c|c|}
\hline & & rabi_maize & $79.30846^{*}$ & 1.31292 & .000 & 74.8158 & 83.8011 \\
\hline & & kharif_maize & $70.05692^{*}$ & 1.31292 & .000 & 65.5643 & 74.5496 \\
\hline & \multirow{5}{*}{ rabi_jowar } & Rabi_rice & $-10.00308^{*}$ & 1.31292 & .000 & -14.4957 & -5.5104 \\
\hline & & Kharif_rice & $-80.80615^{*}$ & 1.31292 & .000 & -85.2988 & -76.3135 \\
\hline & & kharif_jowar & -.07923 & 1.31292 & 1.000 & -4.5719 & 4.4134 \\
\hline & & rabi_maize & -1.49769 & 1.31292 & .933 & -5.9903 & 2.9949 \\
\hline & & kharif_maize & $-10.74923^{*}$ & 1.31292 & .000 & -15.2419 & -6.2566 \\
\hline & \multirow{5}{*}{$\begin{array}{c}\text { kharif_jow } \\
\text { ar }\end{array}$} & Rabi_rice & $-9.92385^{*}$ & 1.31292 & .000 & -14.4165 & -5.4312 \\
\hline & & Kharif_rice & $-80.72692^{*}$ & 1.31292 & .000 & -85.2196 & -76.2343 \\
\hline & & rabi_jowar & .07923 & 1.31292 & 1.000 & -4.4134 & 4.5719 \\
\hline & & rabi_maize & -1.41846 & 1.31292 & .947 & -5.9111 & 3.0742 \\
\hline & & kharif_maize & $-10.67000^{*}$ & 1.31292 & .000 & -15.1626 & -6.1774 \\
\hline & \multirow{5}{*}{ rabi_maize } & Rabi_rice & $-8.50538^{*}$ & 1.31292 & .000 & -12.9980 & -4.0127 \\
\hline & & Kharif_rice & $-79.30846^{*}$ & 1.31292 & .000 & -83.8011 & -74.8158 \\
\hline & & rabi_jowar & 1.49769 & 1.31292 & .933 & -2.9949 & 5.9903 \\
\hline & & kharif_jowar & 1.41846 & 1.31292 & .947 & -3.0742 & 5.9111 \\
\hline & & kharif_maize & $-9.25154^{*}$ & 1.31292 & .000 & -13.7442 & -4.7589 \\
\hline & \multirow{5}{*}{$\begin{array}{c}\text { kharif_mai } \\
\text { ze }\end{array}$} & Rabi_rice & .74615 & 1.31292 & .997 & -3.7465 & 5.2388 \\
\hline & & Kharif_rice & $-70.05692^{*}$ & 1.31292 & .000 & -74.5496 & -65.5643 \\
\hline & & rabi_jowar & $10.74923^{*}$ & 1.31292 & .000 & 6.2566 & 15.2419 \\
\hline & & kharif_jowar & $10.67000^{*}$ & 1.31292 & .000 & 6.1774 & 15.1626 \\
\hline & & rabi_maize & $9.25154^{*}$ & 1.31292 & .000 & 4.7589 & 13.7442 \\
\hline \multirow{20}{*}{ LSD } & \multirow{5}{*}{ Rabi_rice } & Kharif_rice & $-70.80308^{*}$ & 1.31292 & .000 & -73.4203 & -68.1858 \\
\hline & & rabi_jowar & $10.00308^{*}$ & 1.31292 & .000 & 7.3858 & 12.6203 \\
\hline & & kharif_jowar & $9.92385^{*}$ & 1.31292 & .000 & 7.3066 & 12.5411 \\
\hline & & rabi_maize & $8.50538^{*}$ & 1.31292 & .000 & 5.8881 & 11.1226 \\
\hline & & kharif_maize & -.74615 & 1.31292 & .572 & -3.3634 & 1.8711 \\
\hline & & Rabi_rice & $70.80308^{*}$ & 1.31292 & .000 & 68.1858 & 73.4203 \\
\hline & & rabi_jowar & $80.80615^{*}$ & 1.31292 & .000 & 78.1889 & 83.4234 \\
\hline & Kharif_rice & kharif_jowar & $80.72692^{*}$ & 1.31292 & .000 & 78.1097 & 83.3442 \\
\hline & & rabi_maize & $79.30846^{*}$ & 1.31292 & .000 & 76.6912 & 81.9257 \\
\hline & & kharif_maize & $70.05692^{*}$ & 1.31292 & .000 & 67.4397 & 72.6742 \\
\hline & & Rabi_rice & $-10.00308^{*}$ & 1.31292 & .000 & -12.6203 & -7.3858 \\
\hline & & Kharif_rice & $-80.80615^{*}$ & 1.31292 & .000 & -83.4234 & -78.1889 \\
\hline & rabi_jowar & kharif_jowar & -.07923 & 1.31292 & .952 & -2.6965 & 2.5380 \\
\hline & & rabi_maize & -1.49769 & 1.31292 & .258 & -4.1150 & 1.1196 \\
\hline & & kharif_maize & $-10.74923^{*}$ & 1.31292 & .000 & -13.3665 & -8.1320 \\
\hline & & Rabi_rice & $-9.92385^{*}$ & 1.31292 & .000 & -12.5411 & -7.3066 \\
\hline & & Kharif_rice & $-80.72692^{*}$ & 1.31292 & .000 & -83.3442 & -78.1097 \\
\hline & kharif_jow & rabi_jowar & .07923 & 1.31292 & .952 & -2.5380 & 2.6965 \\
\hline & & rabi_maize & -1.41846 & 1.31292 & .284 & -4.0357 & 1.1988 \\
\hline & & kharif_maize & $-10.67000^{*}$ & 1.31292 & .000 & -13.2873 & -8.0527 \\
\hline
\end{tabular}




\begin{tabular}{|c|c|c|c|c|c|c|c|}
\hline & & Rabi_rice & $-8.50538^{*}$ & 1.31292 & .000 & -11.1226 & -5.8881 \\
\hline & & Kharif_rice & $-79.30846^{*}$ & 1.31292 & .000 & -81.9257 & -76.6912 \\
\hline & rabi_maize & rabi_jowar & 1.49769 & 1.31292 & .258 & -1.1196 & 4.1150 \\
\hline & & kharif_jowar & 1.41846 & 1.31292 & .284 & -1.1988 & 4.0357 \\
\hline & & kharif_maize & $-9.25154^{*}$ & 1.31292 & .000 & -11.8688 & -6.6343 \\
\hline & & Rabi_rice & .74615 & 1.31292 & .572 & -1.8711 & 3.3634 \\
\hline & & Kharif_rice & $-70.05692^{*}$ & 1.31292 & .000 & -72.6742 & -67.4397 \\
\hline & 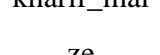 & rabi_jowar & $10.74923^{*}$ & 1.31292 & .000 & 8.1320 & 13.3665 \\
\hline & & kharif_jowar & $10.67000^{*}$ & 1.31292 & .000 & 8.0527 & 13.2873 \\
\hline & & rabi_maize & $9.25154^{*}$ & 1.31292 & .000 & 6.6343 & 11.8688 \\
\hline \multirow{30}{*}{ Bonferroni } & \multirow{5}{*}{ Rabi_rice } & Kharif_rice & $-70.80308^{*}$ & 1.31292 & .000 & -74.7895 & -66.8167 \\
\hline & & rabi_jowar & $10.00308^{*}$ & 1.31292 & .000 & 6.0167 & 13.9895 \\
\hline & & kharif_jowar & $9.92385^{*}$ & 1.31292 & .000 & 5.9374 & 13.9103 \\
\hline & & rabi_maize & $8.50538^{*}$ & 1.31292 & .000 & 4.5190 & 12.4918 \\
\hline & & kharif_maize & -.74615 & 1.31292 & 1.000 & -4.7326 & 3.2403 \\
\hline & \multirow{5}{*}{ Kharif_rice } & Rabi_rice & $70.80308^{*}$ & 1.31292 & .000 & 66.8167 & 74.7895 \\
\hline & & rabi_jowar & $80.80615^{*}$ & 1.31292 & .000 & 76.8197 & 84.7926 \\
\hline & & kharif_jowar & $80.72692^{*}$ & 1.31292 & .000 & 76.7405 & 84.7133 \\
\hline & & rabi_maize & $79.30846^{*}$ & 1.31292 & .000 & 75.3221 & 83.2949 \\
\hline & & kharif_maize & $70.05692^{*}$ & 1.31292 & .000 & 66.0705 & 74.0433 \\
\hline & \multirow{5}{*}{ rabi_jowar } & Rabi_rice & $-10.00308^{*}$ & 1.31292 & .000 & -13.9895 & -6.0167 \\
\hline & & Kharif_rice & $-80.80615^{*}$ & 1.31292 & .000 & -84.7926 & -76.8197 \\
\hline & & kharif_jowar & -.07923 & 1.31292 & 1.000 & -4.0656 & 3.9072 \\
\hline & & rabi_maize & -1.49769 & 1.31292 & 1.000 & -5.4841 & 2.4887 \\
\hline & & kharif_maize & $-10.74923^{*}$ & 1.31292 & .000 & -14.7356 & -6.7628 \\
\hline & \multirow{5}{*}{$\begin{array}{c}\text { kharif_jow } \\
\text { ar }\end{array}$} & Rabi_rice & $-9.92385^{*}$ & 1.31292 & .000 & -13.9103 & -5.9374 \\
\hline & & Kharif_rice & $-80.72692^{*}$ & 1.31292 & .000 & -84.7133 & -76.7405 \\
\hline & & rabi_jowar & .07923 & 1.31292 & 1.000 & -3.9072 & 4.0656 \\
\hline & & rabi_maize & -1.41846 & 1.31292 & 1.000 & -5.4049 & 2.5679 \\
\hline & & kharif_maize & $-10.67000^{*}$ & 1.31292 & .000 & -14.6564 & -6.6836 \\
\hline & \multirow{5}{*}{ rabi_maize } & Rabi_rice & $-8.50538^{*}$ & 1.31292 & .000 & -12.4918 & -4.5190 \\
\hline & & Kharif_rice & $-79.30846^{*}$ & 1.31292 & .000 & -83.2949 & -75.3221 \\
\hline & & rabi_jowar & 1.49769 & 1.31292 & 1.000 & -2.4887 & 5.4841 \\
\hline & & kharif_jowar & 1.41846 & 1.31292 & 1.000 & -2.5679 & 5.4049 \\
\hline & & kharif_maize & $-9.25154^{*}$ & 1.31292 & .000 & -13.2379 & -5.2651 \\
\hline & \multirow{5}{*}{$\begin{array}{c}\text { kharif_mai } \\
\text { ze }\end{array}$} & Rabi_rice & .74615 & 1.31292 & 1.000 & -3.2403 & 4.7326 \\
\hline & & Kharif_rice & $-70.05692^{*}$ & 1.31292 & .000 & -74.0433 & -66.0705 \\
\hline & & rabi_jowar & $10.74923^{*}$ & 1.31292 & .000 & 6.7628 & 14.7356 \\
\hline & & kharif_jowar & $10.67000^{*}$ & 1.31292 & .000 & 6.6836 & 14.6564 \\
\hline & & rabi_maize & $9.25154^{*}$ & 1.31292 & .000 & 5.2651 & 13.2379 \\
\hline
\end{tabular}




\begin{tabular}{|c|c|c|c|c|c|c|c|}
\hline \multirow{5}{*}{ 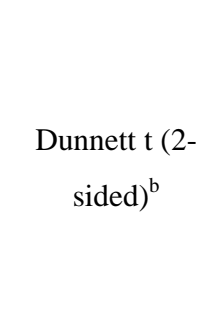 } & Rabi_rice & kharif_maize & -.74615 & 1.31292 & .970 & -4.1210 & 2.6287 \\
\hline & Kharif_rice & kharif_maize & $70.05692^{*}$ & 1.31292 & .000 & 66.6821 & 73.4317 \\
\hline & rabi_jowar & kharif_maize & $-10.74923^{*}$ & 1.31292 & .000 & -14.1240 & -7.3744 \\
\hline & $\begin{array}{c}\text { kharif_jow } \\
\text { ar }\end{array}$ & kharif_maize & $-10.67000^{*}$ & 1.31292 & .000 & -14.0448 & -7.2952 \\
\hline & rabi_maize & kharif_maize & $-9.25154^{*}$ & 1.31292 & .000 & -12.6263 & -5.8767 \\
\hline \multicolumn{8}{|c|}{ The mean difference is significant at the 0.05 level. } \\
\hline
\end{tabular}

\subsection{Homogenous Subsets}

Table 5:Homogeneous Subsets rabi_rice

\begin{tabular}{|c|c|c|c|c|c|}
\hline & \multirow[t]{2}{*}{ target } & \multirow[t]{2}{*}{$\mathbf{N}$} & \multicolumn{3}{|c|}{ Subset for alpha $=\mathbf{0 . 0 5}$} \\
\hline & & & 1 & 2 & 3 \\
\hline \multirow{7}{*}{$\begin{array}{c}\text { Tukey } \\
\text { HSD }\end{array}$} & rabi_jowar & 13 & 3.2062 & & \\
\hline & kharif_jowar & 13 & 3.2854 & & \\
\hline & rabi_maize & 13 & 4.7038 & & \\
\hline & Rabi_rice & 13 & & 13.2092 & \\
\hline & kharif_maize & 13 & & 13.9554 & \\
\hline & Kharif_rice & 13 & & & 84.0123 \\
\hline & Sig. & & .863 & .993 & 1.000 \\
\hline \multirow{7}{*}{ Scheffe } & rabi_jowar & 13 & 3.2062 & & \\
\hline & kharif_jowar & 13 & 3.2854 & & \\
\hline & rabi_maize & 13 & 4.7038 & & \\
\hline & Rabi_rice & 13 & & 13.2092 & \\
\hline & kharif_maize & 13 & & 13.9554 & \\
\hline & Kharif_rice & 13 & & & 84.0123 \\
\hline & Sig. & & .933 & .997 & 1.000 \\
\hline \multicolumn{6}{|c|}{ Means for groups in homogeneous subsets are displayed. } \\
\hline
\end{tabular}

The homogenous subsets output is produced by a request for post hoc test and addresses the same questions as the multiple comparisons table for post hoc analysis i.e. which pair of groups have significantly different means on the dependent variable.

\subsection{Means Plot}

Means plot figure is used to see if the mean varies between different groups of the data. In the above sample plot different crops with different seasons provide the grouping. A mean plot can then be generated with these groups to see if the mean is increasing or decreasing over the time. This sample plot shows a shift of location after Kharif rice.

Also, linear regression has been fitted for three crops and it has been found that during Rabi season the production of Rice and Jowar seem to be the same during this season. Maize has a significant production indicating that a change when compared to Rice and Jowar, while during Kharif there is a clear cut significant production for all the three crops that is Rice, Jowar and Maize. Thus, this analysis indicates a significant pattern during Kharif and a different pattern during Rabi seasons.

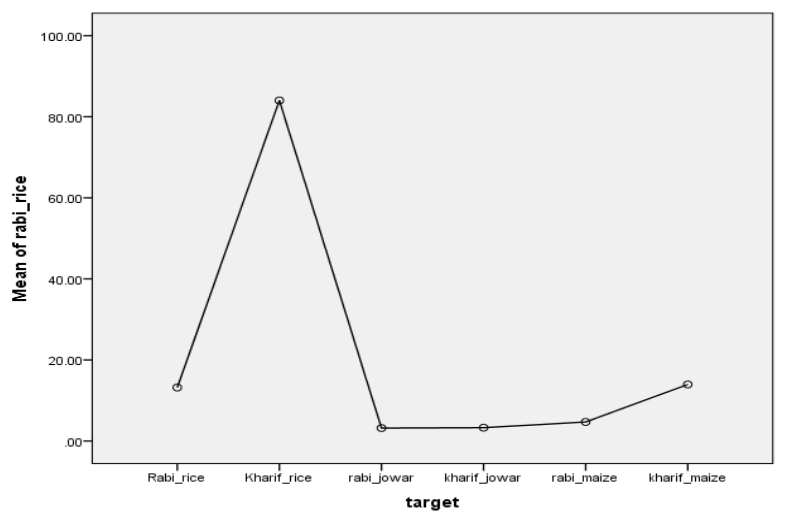

Fig1: Means Plot

\subsection{Regression Analysis}

\subsubsection{Regression Analysis for rabi_rice}

Table 6: Variables Entered/Removed

\begin{tabular}{|c|c|c|c|}
\hline Model & $\begin{array}{c}\text { Variables } \\
\text { Entered }\end{array}$ & Variables Removed & Method \\
\hline 1 & Year &. & Enter \\
\hline
\end{tabular}

a. Dependent Variable: rabi_rice.

b. All requested variables entered.

Table 7: Model Summary

\begin{tabular}{|c|c|c|c|c|}
\hline Model & $\mathrm{R}$ & R Square & $\begin{array}{c}\text { Adjusted R } \\
\text { Square }\end{array}$ & $\begin{array}{c}\text { Std. Error } \\
\text { of the } \\
\text { Estimate }\end{array}$ \\
\hline 1 & .515 & .265 & .199 & 1.36585 \\
\hline
\end{tabular}

a. Predictors: (Constant), year

Table8: ANOVA

\begin{tabular}{|c|c|c|c|c|c|c|}
\hline \multicolumn{2}{|c|}{ Model } & $\begin{array}{c}\text { Sum of } \\
\text { Squares }\end{array}$ & df & $\begin{array}{c}\text { Mean } \\
\text { Square }\end{array}$ & F & Sig. \\
\hline & Regression & 7.417 & 1 & 7.417 & 3.976 & .072 \\
\hline
\end{tabular}




\begin{tabular}{|l|c|c|c|c|c|c|}
\hline & Residual & 20.521 & 11 & 1.866 & & \\
\hline & Total & 27.938 & 12 & & & \\
\hline
\end{tabular}

a. Dependent Variable: rabi_rice

b. Predictors: (Constant), year

\section{Table 9 :Coefficients}

\begin{tabular}{|c|c|c|c|c|c|}
\hline Model & \multicolumn{2}{|c|}{$\begin{array}{c}\text { Unstandardized } \\
\text { Coefficients }\end{array}$} & $\begin{array}{c}\text { Standardized } \\
\text { Coefficients }\end{array}$ & $\mathrm{t}$ & Sig. \\
\hline & $\mathrm{B}$ & $\begin{array}{c}\text { Std. } \\
\text { Error }\end{array}$ & Beta & & \\
\hline Constant & -392.344 & 203.399 & & -1.929 & .080 \\
\hline year & .202 & .101 & .515 & 1.994 & .072 \\
\hline
\end{tabular}

a. Dependent Variable: rabi_rice

\subsubsection{Regression Analysis for kharif_rice}

Table 10: Variables Entered/Removed

\begin{tabular}{|c|c|c|c|}
\hline Model & $\begin{array}{c}\text { Variables } \\
\text { Entered }\end{array}$ & $\begin{array}{c}\text { Variables } \\
\text { Removed }\end{array}$ & Method \\
\hline 1 & year & . & Enter \\
\hline
\end{tabular}

a) Dependent Variable: kharif_rice

b) All requested variables entered.

Table 11: Model Summary

\begin{tabular}{|c|c|c|c|c|}
\hline Model & $\mathrm{R}$ & $\mathrm{R}$ Square & $\begin{array}{c}\text { Adjusted R } \\
\text { Square }\end{array}$ & $\begin{array}{c}\text { Std. Error of } \\
\text { the Estimate }\end{array}$ \\
\hline 1 & .830 & .688 & .660 & 4.11200 \\
\hline
\end{tabular}

a. Predictors: (Constant), year

Table 12: ANOVA

\begin{tabular}{|c|c|c|c|c|c|c|}
\hline \multicolumn{2}{|c|}{ Model } & $\begin{array}{c}\text { Sum of } \\
\text { Squares }\end{array}$ & df & $\begin{array}{c}\text { Mean } \\
\text { Square }\end{array}$ & F & Sig. \\
\hline \multirow{2}{*}{} & Regression & 411.061 & 1 & 411.061 & 24.11 & .000 \\
\cline { 2 - 7 } & Residual & 185.994 & 11 & 16.909 & & \\
\cline { 2 - 7 } & Total & 597.055 & 12 & & & \\
\hline
\end{tabular}

a) Dependent Variable: kharif_rice

b) Predictors: (Constant), year

Table 13 :Coefficients

\begin{tabular}{|c|c|c|c|c|c|}
\hline \multirow{2}{*}{ Model } & \multicolumn{2}{|c|}{$\begin{array}{c}\text { Unstandardized } \\
\text { Coefficients }\end{array}$} & $\begin{array}{c}\text { Standardized } \\
\text { Coefficients }\end{array}$ & \multirow{2}{*}{ Sig. } \\
\cline { 2 - 5 } & $\mathrm{B}$ & $\begin{array}{c}\text { Std. } \\
\text { Error }\end{array}$ & Beta & & \\
\hline Constant & -2935.228 & 612.347 & & 4.793 & .001 \\
\hline year & 1.503 & .305 & .830 & 4.931 & .000 \\
\hline
\end{tabular}

a. Dependent Variable: kharif_rice

3.6.3 Regression Analysis for rabi_jowar Table 14: Variables Entered/Removed

\begin{tabular}{|c|c|c|c|}
\hline Model & $\begin{array}{c}\text { Variables } \\
\text { Entered }\end{array}$ & Variables Removed & Method \\
\hline 1 & Year & . & Enter \\
\hline
\end{tabular}

a) Dependent Variable: rabi_jowar.

b) All requested variables entered

Table 15: Model Summary

\begin{tabular}{|c|c|c|c|c|}
\hline Model & $\mathrm{R}$ & $\mathrm{R}$ Square & $\begin{array}{c}\text { Adjusted R } \\
\text { Square }\end{array}$ & $\begin{array}{c}\text { Std. Error } \\
\text { of the } \\
\text { Estimate }\end{array}$ \\
\hline 1 & .81 & .007 & .084 & 0.68026 \\
\hline
\end{tabular}

b. Predictors: (Constant), year

Table 16: ANOVA

\begin{tabular}{|c|c|c|c|c|c|}
\hline Model & $\begin{array}{c}\text { Sum of } \\
\text { Squares }\end{array}$ & df & $\begin{array}{c}\text { Mean } \\
\text { Square }\end{array}$ & F & Sig. \\
\hline Regression & 0.34 & 1 & .034 & .073 & .792 \\
\hline Residual & 5.090 & 11 & .463 & & \\
\hline Total & 5.124 & 12 & & & \\
\hline
\end{tabular}

a) Dependent Variable: rabi_jowar

b) Predictors: (Constant), year

Table 17: Coefficients

\begin{tabular}{|c|c|c|c|c|c|}
\hline \multirow{2}{*}{ Model } & \multicolumn{2}{|c|}{$\begin{array}{c}\text { Unstandardized } \\
\text { Coefficients }\end{array}$} & $\begin{array}{c}\text { Standardized } \\
\text { Coefficients }\end{array}$ & $\mathrm{t}$ & Sig. \\
\cline { 2 - 4 } & $\mathrm{B}$ & $\begin{array}{c}\text { Std. } \\
\text { Error }\end{array}$ & Beta & & \\
\hline Constant & 30.582 & 101.303 & & .302 & .768 \\
\hline year & -0.014 & .050 & .081 & .270 & .792 \\
\hline
\end{tabular}

a. Dependent Variable: rabi_jowar

3.6.4 Regression Analysis for kharif_jowar

Table 18: Variables Entered/Removed

\begin{tabular}{|c|c|c|c|}
\hline Model & $\begin{array}{c}\text { Variables } \\
\text { Entered }\end{array}$ & Variables Removed & Method \\
\hline 1 & Year &. & Enter \\
\hline
\end{tabular}

a. Dependent Variable: kharif_jowar.

b. All requested variables entered 
Table 19: Model Summary

\begin{tabular}{|c|c|c|c|c|}
\hline Model & $\mathrm{R}$ & R Square & $\begin{array}{c}\text { Adjusted R } \\
\text { Square }\end{array}$ & $\begin{array}{c}\text { Std. Error } \\
\text { of the } \\
\text { Estimate }\end{array}$ \\
\hline 1 & .927 & .860 & .847 & 0.33429 \\
\hline
\end{tabular}

c. Predictors: (Constant), year

Table 20: ANOVA

\begin{tabular}{|c|c|c|c|c|c|}
\hline Model & $\begin{array}{c}\text { Sum of } \\
\text { Squares }\end{array}$ & df & $\begin{array}{c}\text { Mean } \\
\text { Square }\end{array}$ & F & Sig. \\
\hline Regression & 7.563 & 1 & 7.563 & 67.676 & .000 \\
\hline Residual & 1.229 & 11 & .112 & & \\
\hline Total & 8.792 & 12 & & & \\
\hline
\end{tabular}

a) Dependent Variable: kharif_jowar

b) Predictors: (Constant), year

Table 21: Coefficients

\begin{tabular}{|c|c|c|c|c|c|}
\hline \multirow{2}{*}{ Model } & \multicolumn{2}{|c|}{$\begin{array}{c}\text { Unstandardized } \\
\text { Coefficients }\end{array}$} & $\begin{array}{c}\text { Standardized } \\
\text { Coefficients }\end{array}$ & \multirow{2}{*}{ Sig. } \\
\cline { 2 - 5 } & B & $\begin{array}{c}\text { Std. } \\
\text { Error }\end{array}$ & Beta & & \\
\hline Constant & 412.812 & 49.781 & & 8.293 & .000 \\
\hline year & -.204 & .025 & -.927 & -8.227 & .000 \\
\hline
\end{tabular}

a. Dependent Variable: kharif_jowar

3.6.4 Regression Analysis for maize_rabi Table 22: Variables Entered/Removed

\begin{tabular}{|c|c|c|c|}
\hline Model & $\begin{array}{c}\text { Variables } \\
\text { Entered }\end{array}$ & Variables Removed & Method \\
\hline 1 & Year &. & Enter \\
\hline
\end{tabular}

a) Dependent Variable: maize_rabi

b) All requested variables entered.

Table 23: Model Summary

\begin{tabular}{|c|c|c|c|c|}
\hline Model & $\mathrm{R}$ & R Square & $\begin{array}{c}\text { Adjusted R } \\
\text { Square }\end{array}$ & $\begin{array}{c}\text { Std. Error } \\
\text { of the } \\
\text { Estimate }\end{array}$ \\
\hline 1 & .912 & .832 & .816 & 0.70511 \\
\hline
\end{tabular}

d. Predictors: (Constant), year

Table 24: ANOVA

\begin{tabular}{|c|c|c|c|c|c|}
\hline Model & $\begin{array}{c}\text { Sum of } \\
\text { Squares }\end{array}$ & df & $\begin{array}{c}\text { Mean } \\
\text { Square }\end{array}$ & F & Sig. \\
\hline
\end{tabular}

\begin{tabular}{|c|c|c|c|c|c|c|}
\hline & Regression & 27.000 & 1 & 27.000 & 53.306 & .000 \\
\hline Residual & 5.469 & 11 & .497 & & \\
\hline Total & 32.469 & 12 & & & \\
\hline
\end{tabular}

a) Dependent Variable: maize_rabi

b) Predictors: (Constant), year

Table 25 : Coefficients

\begin{tabular}{|c|c|c|c|c|c|}
\hline Model & \multicolumn{2}{|c|}{$\begin{array}{c}\text { Unstandardized } \\
\text { Coefficients }\end{array}$} & $\begin{array}{c}\text { Standardized } \\
\text { Coefficients }\end{array}$ & \multirow{2}{*}{ Sig. } \\
\cline { 2 - 4 } & $\mathrm{B}$ & $\begin{array}{c}\text { Std. } \\
\text { Error }\end{array}$ & Beta & & \\
\hline Constant & -769.092 & 105.004 & & -7.324 & .000 \\
\hline year & .385 & .052 & .912 & -7.369 & .000 \\
\hline
\end{tabular}

a. Dependent Variable: maize_rabi.

\subsubsection{Regression Analysis for maize_kharif}

Table 26: Variables Entered/Removed

\begin{tabular}{|c|c|c|c|}
\hline Model & $\begin{array}{c}\text { Variables } \\
\text { Entered }\end{array}$ & Variables Removed & Method \\
\hline 1 & Year & $\cdot$ & Enter \\
\hline
\end{tabular}

a) Dependent Variable: maize_kharif

b) All requested variables entered

Table 27: Model Summary

\begin{tabular}{|c|c|c|c|c|}
\hline Model & $\mathrm{R}$ & R Square & $\begin{array}{c}\text { Adjusted R } \\
\text { Square }\end{array}$ & $\begin{array}{c}\text { Std. Error } \\
\text { of the } \\
\text { Estimate }\end{array}$ \\
\hline 1 & .710 & .503 & .458 & 2.47175 \\
\hline
\end{tabular}

e. Predictors: (Constant), year

Table 28: ANOVA

\begin{tabular}{|c|c|c|c|c|c|}
\hline Model & $\begin{array}{c}\text { Sum of } \\
\text { Squares }\end{array}$ & df & $\begin{array}{c}\text { Mean } \\
\text { Square }\end{array}$ & F & Sig. \\
\hline Regression & 68.138 & 1 & 68.138 & 11.153 & .007 \\
\hline Residual & 67.205 & 11 & 6.110 & & \\
\hline Total & 135.343 & 12 & & & \\
\hline
\end{tabular}

a) Dependent Variable: maize_kharif

b) . Predictors: (Constant), year 
Table 29: Coefficients

\begin{tabular}{|c|c|c|c|c|c|}
\hline \multirow{2}{*}{ Model } & \multicolumn{2}{|c|}{$\begin{array}{c}\text { Unstandardized } \\
\text { Coefficients }\end{array}$} & $\begin{array}{c}\text { Standardized } \\
\text { Coefficients }\end{array}$ & \multirow{2}{*}{ Sig. } \\
\cline { 2 - 5 } & B & $\begin{array}{c}\text { Std. } \\
\text { Error }\end{array}$ & Beta & & \\
\hline Constant & -1215.28 & 368.086 & & -3.302 & .007 \\
\hline year & .612 & .183 & .710 & -3.340 & .007 \\
\hline
\end{tabular}

a. Dependent Variable: maize_kharif.

b. Dependent Variable: maize_kharif.

For Regression analysis, for instance if we take maize_kharif in Model summary table (table 27), the table provides $\mathrm{R}$ and $R^{2}$ values. The $R$ value represents the simple correlation and is 0.710 , which indicates a high degree of Correlation, the $\mathrm{R}^{2}$ value indicates how much of the total variation in the dependent variable(production for maize_kharif) can be explained by the independent variable(year). In this case $\mathrm{R}^{2}$ is 0.503 i.e. $50.3 \%$ can be explained, which is large. Increase in $\mathrm{R}^{2}$ value means increase in the model's fit is statistically significant.

The next table is ANOVA table (table 28)which report how well the regression equation fit the data (i.e. predict the dependent variable). In this table the significance is $0.007>0.05$ indicates that overall the regression model is not statistically significant .The coefficient table (table 29)provides us with necessary information to predict production from year as well as determine whether year contributes statistical significance to the model, it also shows the regression coefficients, intercept and the significance of all coefficients .It is found that linear regression analysis estimate the linear regression function to be $\mathrm{y}$ (production) $=$ $1215.288+0.612 * x($ year $)$.

\section{CONCLUSION}

The significant value ( $\mathrm{p}$ value) for each term tests the null hypothesis that the coefficient is equal to zero (no effect). A low p-value $(<0.05)$ indicates the rejection of the null hypothesis. In other words a predictor that has a low p-value is likely to be a meaningful addition to this model because changes in the predictor's value are related to changes in the response variable. Conversely a larger (insignificant) $p$-value suggests that changes in the predictor are not associated with changes in the response (dependent variable). The conclusion drawn can help in the future prediction of production of three major crops.

\section{REFERENCES}

[1] Mann, Prem S (1995) Introductory Statistics ( $2^{\text {nd }}$ edition) Wiley.

[2] Trochim, William M.K (2006),"Descriptive Statistics", Research Methods Knowledge Base.

[3] Jaccard. J; Becker, M.A.Wood.G (1984). "Pair wise multiple comparison procedures: A review".

[4] Bailey R.A (2008) "Design of comparative experiments". Cambridge University Press.

[5] Cochran, William G; Cox, Gertrude M (1992) Experimental Designs ( $2^{\text {nd }}$ edition) New York: Wiley.

[6] Ronald A Fisher(1954) Statistical Methods for Research Workers(twelfth edition)

[7] Ajit Kaur Bhatia, Rajender Prasad, S.K Shama and Rajinder Kaur (2005), Statistical assessment of different crop rotations, Journal of Farming Systems Research \& Development, 11 (2), 190-196.

[8] J S Dhekale, Rajendar Prasad and V K Gupta (2003), Analysis of intercropping experiments using experiments with mixtures methodology. Journal of Indian Society of Agricultural Statistics. 56 (3), 260-266. 